\title{
On the Geometry and Quantization of Symplectic Howe Pairs
}

\author{
Carsten Balleier* \\ Tilmann Wurzbacher
}

Laboratoire de Mathématiques et Applications de Metz

Université Paul Verlaine-Metz et C. N. R.S.

Ile du Saulcy, F-57045 Metz, France

wurzbacher@math .univ-metz.fr

October 5, 2009

MSC: Primary 53D20 $\cdot$ 53D50; Secondary 17B08 $\cdot 32 \mathrm{Q} 15 \cdot 57 \mathrm{~S} 15$

\begin{abstract}
We study the orbit structure and the geometric quantization of a pair of mutually commuting hamiltonian actions on a symplectic manifold. If the pair of actions fulfils a symplectic Howe condition, we show that there is a canonical correspondence between the orbit spaces of the respective moment images. Furthermore, we show that reduced spaces with respect to the action of one group are symplectomorphic to coadjoint orbits of the other group. In the Kähler case we show that the linear representation of a pair of compact Lie groups on the geometric quantization of the manifold is then equipped with a representationtheoretic Howe duality.
\end{abstract}

\section{Introduction}

In representation theory, Howe duality ([How89]) is well-known. In the model situation of a product $G_{1} \times G_{2}$ of two compact connected Lie groups linearly represented on a finite dimensional complex vector space $U\left(\varrho: G_{1} \times G_{2} \rightarrow G L(U)\right)$, we say that the representation satisfies the Howe condition or is equipped with a Howe duality if there is a subset $\mathcal{D}$ of $\widehat{G_{1}}$, the set of equivalence classes of

*supported by the German Research Foundation (DFG) and the Université franco-allemande (DFHUFA) via the IRTG 1133 "Geometry and Analysis of Symmetries" 
irreducible complex representations of $G_{1}$, and an injective map $\Lambda: \mathcal{D} \rightarrow \widehat{G_{2}}$ such that

$$
U \cong \bigoplus_{\alpha \in \mathcal{D}} V_{\alpha} \otimes W_{\Lambda(\alpha)}
$$

where $V_{\alpha}$ represents a class $\alpha$ in $\mathcal{D}$ and $W_{\Lambda(\alpha)}$ represents the class $\Lambda(\alpha)$. Denoting for $k=1,2$ the restriction of the representation $\varrho$ to $G_{k}$ by $\varrho_{k}$ (as well as the corresponding Lie algebra representation), condition $(*)$ is equivalent to

$$
(* *) \quad Z_{\operatorname{End}(U)}\left(\varrho_{i}\left(\mathcal{U} \mathfrak{g}_{i}\right)\right)=\varrho_{j}\left(\mathcal{U} \mathfrak{g}_{j}\right) \quad \text { for } i \neq j,
$$

where $\mathcal{U} \mathfrak{g}$ is the universal enveloping algebra (over $\mathbb{C}$ ) of a Lie algebra $\mathfrak{g}$, and for a set $S \subseteq \operatorname{End}(U), Z_{\operatorname{End}(U)}(S)$ is the centralizer of $S$ in the endomorphisms of $U$. This equivalence is easily shown using standard facts from representation theory, as found, e.g., in [GW98]. Obviously, condition (*) can be formulated for representations on a complex vector space $U$ having countable dimension and -upon passing to an appropriate completion on the right hand side- for representations on Fréchet or more general topological vector spaces.

In the spirit of quantization/dequantization of physical and mathematical structures (going back to the correspondence principle of Niels Bohr, see [RN76]), attempts to find analogous notions in symplectic geometry have been made. This concerns analogues of commutants ( $G_{\mathscr{V}}$ in [KKS78], polar actions in [OR04]) and dual pair notions (as in [Wei83]), as well as the study of the orbit structure in particular cases like in, e.g., [Ada87]. In this article, we study the setting of commuting hamiltonian (proper) actions of two Lie groups on a symplectic manifold. We define in this situation a symplectic Howe condition, naturally corresponding to the above condition $(* *)$, namely

$$
Z_{C^{\infty}(M)}\left(\Phi_{i}^{*} C^{\infty}\left(\mathfrak{g}_{i}^{*}\right)\right)=\Phi_{j}^{*} C^{\infty}\left(\mathfrak{g}_{j}^{*}\right) \quad \text { for } i \neq j .
$$

Here and in the sequel, the Lie algebra of a Lie group $G$ will always be denoted by $\mathfrak{g}$ and, for $A \subseteq C^{\infty}(M), Z_{C^{\infty}(M)}(A)=\left\{f \in C^{\infty}(M) \mid\{f, a\}=0 \forall a \in A\right\}$.

We analyse thoroughly its consequences on the orbit structure, obtaining results that refine those of [KKS78]. We then prove an orbit correspondence and show the conservation of integrality under the correspondence. Furthermore we show that the Marsden-Weinstein reduced spaces of the action of one group are equivariantly symplectomorphic to coadjoint orbits in the moment image of the other group action. To this setting we finally apply geometric quantization and obtain in the Kähler case a decomposition of the quantization of the initial symplectic manifold as in $(*)$. That is, given a pair of commuting actions by holomorphic transformation groups on a Kähler manifold satisfying the symplectic Howe condition, the linear action of the pair on the holomorphic quantization (the holomorphic sections of the quantizing holomorphic line bundle) satisfies the representation-theoretic Howe condition. In the last section we illustrate the results with some simple but instructive examples. 


\section{Properties of Commuting Hamiltonian Ac- tions}

The setting throughout this article is a smooth connected symplectic manifold $(M, \omega)$ on which two smooth actions of the Lie groups $G_{1}$ and $G_{2}$ are given.

Assume these actions commute and are hamiltonian such that each action admits an equivariant moment map $\Phi_{i}: M \rightarrow \mathfrak{g}_{i}^{*}(i=1,2)$, i. e., they satisfy $\mathrm{d} \Phi_{i}^{\xi}=\omega\left(\tau_{i}(\xi), \cdot\right)$ and $\Phi_{i}(g \cdot z)=\operatorname{Ad}^{*}(g) \Phi_{i}(z) \forall z \in M, g \in G_{i}$. Here, for any $\xi \in \mathfrak{g}_{i}$, we denote by $\tau_{i}(\xi)$ the fundamental vector field on $M$ associated to $\xi$ and by $\Phi_{i}^{\xi}$ the component of the $i$-moment map in direction $\xi$. We first notice the following easy lemma.

Lemma 2.1. Let $(M, \omega)$ be a symplectic manifold. Let the Lie groups $G_{1}$ and $G_{2}$ act symplectically on $M$, admitting equivariant moment maps $\Phi_{1}$ and $\Phi_{2}$. Assume these actions commute. Then the product group $G_{1} \times G_{2}$ acts symplectically on $M$ with an equivariant moment map, and notably for any $\xi \in \mathfrak{g}_{1}$ and any $\eta \in \mathfrak{g}_{2}$ the Poisson bracket of the moment components vanishes, i.e.,

$$
\left\{\Phi_{1}^{\xi}, \Phi_{2}^{\eta}\right\}=0
$$

Proof. Obvious.

In other words, the moment map of the first action is constant along the connected components of the orbits of the second action, and vice versa. For $G_{1}$ and $G_{2}$ (or, at least, their orbits) connected, this can be rephrased as

$$
\Phi_{i}^{*} C^{\infty}\left(\mathfrak{g}_{i}^{*}\right) \subseteq C^{\infty}(M)^{G_{j}} \quad \text { for } i \neq j .
$$

Here we observe the inclusion of the collective functions for the first action in the set of invariants for the second action, and vice versa. Let us now observe the following useful -and most certainly folkloristic- result.

Lemma 2.2. Let $G$ be a connected Lie group and $(M, \omega)$ a symplectic manifold. If $G$ acts symplectically with an equivariant moment map $\Phi$ on $M$, then

$$
Z_{C^{\infty}(M)}\left(\Phi^{*} C^{\infty}\left(\mathfrak{g}^{*}\right)\right)=C^{\infty}(M)^{G} .
$$

Proof. Note that for $f \in C^{\infty}(M)$ and $\xi \in \mathfrak{g}$, one has that $\xi^{M}(f)=\left\{f, \Phi^{\xi}\right\}$ (where $\xi^{M}=\tau(\xi)$ denotes the fundamental vector field on $M$ associated to $\xi \in \mathfrak{g}$ ), and, furthermore, if $z \in M$, one has $\left\{f, \Phi^{\xi}\right\}(z)=-\left\langle\xi, T_{z} \Phi\left(X_{f \mid z}\right)\right\rangle$ where $\langle\cdot, \cdot\rangle$ is the natural pairing between $\mathfrak{g}$ and $\mathfrak{g}^{*}$ and $\omega\left(X_{f}, \cdot\right)=\mathrm{d} f$, i. e., $X_{f}$ is the hamiltonian vector field associated to $f$.

Given now $f \in C^{\infty}(M)^{G}$ one has that for all $z \in M, T_{z} \Phi\left(X_{f \mid z}\right)=0$. If $h \in C^{\infty}\left(\mathfrak{g}^{*}\right)$ and $z \in M$, we have

$$
\{h \circ \Phi, f\}(z)=\mathrm{d}(h \circ \Phi)_{z}\left(X_{f \mid z}\right)=\mathrm{d} h_{\Phi(z)}\left(T_{z} \Phi\left(X_{f \mid z}\right)\right)=0 .
$$

On the other hand, if $f \in Z_{C^{\infty}(M)}\left(\Phi^{*} C^{\infty}\left(\mathfrak{g}^{*}\right)\right)$ and $\xi \in \mathfrak{g}$, then $\xi^{M}(f)=$ $\left\{f, \Phi^{\xi}\right\}=0$, and thus by the connectedness of $G, f \in C^{\infty}(M)^{G}$. 
Remark 2.3. More subtle relations between centralizers of pullbacks via $\Phi$ and invariant functions are shown in [KL97] in the case that $G$ is compact (often extending, in fact, to proper actions).

With the two preceding lemmata we immediately get that

$$
\Phi_{i}^{*} C^{\infty}\left(\mathfrak{g}_{i}^{*}\right) \subseteq Z_{C^{\infty}(M)}\left(\Phi_{j}^{*} C^{\infty}\left(\mathfrak{g}_{j}^{*}\right)\right) \quad \text { for } i \neq j
$$

Equality of the respective sets is obviously the classical analogue of $(* *)$ upon interpreting $\Phi^{*} C^{\infty}\left(\mathfrak{g}^{*}\right)$ as the "classical collective observables (w. r. t. to a hamiltonian $G$-action on $M)$ " and $\varrho(\mathcal{U} \mathfrak{g})$ as the "quantum collective observables (w.r.t. to a linear $G$-representation)". Therefore, it is natural to study the following situation.

Definition 2.4. We say that two commuting hamiltonian actions satisfy the symplectic Howe condition or form a symplectic Howe pair if

$$
Z_{C^{\infty}(M)}\left(\Phi_{i}^{*} C^{\infty}\left(\mathfrak{g}_{i}^{*}\right)\right)=\Phi_{j}^{*} C^{\infty}\left(\mathfrak{g}_{j}^{*}\right) \quad \text { for } i \neq j
$$

is satisfied.

Remark 2.5. The above condition is clearly close to the notions of dual pairs presented in Ch. 11 of [OR04]. However, we do not require the moment maps to be submersions nor surjections when dealing with the symplectic Howe condition.

Assuming further the properness of both actions, the symplectic Howe condition has the following important consequence for the orbit structure of these commuting actions.

Proposition 2.6. Let commuting hamiltonian proper actions of the connected Lie groups $G_{1}$ and $G_{2}$ with equivariant moment maps $\Phi_{1}$ and $\Phi_{2}$ be given on the symplectic manifold $(M, \omega)$. Then the symplectic Howe condition

$$
Z_{C^{\infty}(M)}\left(\Phi_{i}^{*} C^{\infty}\left(\mathfrak{g}_{i}^{*}\right)\right)=\Phi_{j}^{*} C^{\infty}\left(\mathfrak{g}_{j}^{*}\right) \quad \text { for } i \neq j
$$

implies that

$$
\forall z \in M \text { holds } \Phi_{i}^{-1}\left(\Phi_{i}(z)\right)=G_{j} \cdot z \quad \text { for } i \neq j,
$$

i. e., the levels sets of the moment maps of one action are the orbits of the other one.

Proof. Let $i, j \in\{1,2\}$ such that $i+j=3$. Fix $z \in M$ and denote $\Phi_{i}^{-1}\left(\Phi_{i}(z)\right)=$ $N_{z}$. By Lemma 2.1 and the connectedness of $G_{j}$, the $G_{j}$-action on $M$ preserves the subset $N_{z} \subseteq M$.

Assume that $N_{z} \neq G_{j} \cdot z$. Then there exists $z^{\prime} \in N_{z}$ such that $G_{j} \cdot z^{\prime} \cap G_{j} \cdot z=\emptyset$. Since the $G_{j}$-action is proper, there exists $f \in C^{\infty}(M)^{G_{j}}$ such that $f\left(z^{\prime}\right) \neq f(z)$ (separation of orbits by invariant functions follows easily from the slice theorem for proper actions). 
By Lemma 2.2 and the symplectic Howe condition, we have

$$
C^{\infty}(M)^{G_{j}}=Z_{C^{\infty}(M)}\left(\Phi_{j}^{*} C^{\infty}\left(\mathfrak{g}_{j}^{*}\right)\right)=\Phi_{i}^{*} C^{\infty}\left(\mathfrak{g}_{i}^{*}\right),
$$

i. e., there is a smooth function $h: \mathfrak{g}_{i}^{*} \rightarrow \mathbb{R}$ such that $f=h \circ \Phi_{i}$. Thus we arrive at $f\left(z^{\prime}\right)=h\left(\Phi_{i}\left(z^{\prime}\right)\right)=h\left(\Phi_{i}(z)\right)=f(z)$, contradicting our assumption. Consequently, $\Phi_{i}^{-1}\left(\Phi_{i}(z)\right)=G_{j} \cdot z$.

Knowing the level sets of the moment maps as precisely as above permits to relate the stabilizers of the actions on $M$ and on the moment images $\Phi_{i}(M)$.

Lemma 2.7. Let $G_{1}$ and $G_{2}$ be Lie groups and $(M, \omega)$ be a symplectic manifold. Let hamiltonian actions of both groups on $M$ be given which commute, and denote the equivariant moment maps by $\Phi_{i}: M \rightarrow \mathfrak{g}_{i}^{*}$. Let $G_{12, z}=\left\{\left(g, g^{\prime}\right) \in G_{1} \times\right.$ $\left.G_{2} \mid\left(g, g^{\prime}\right) \cdot z=z\right\}$ be the stabilizer of a point $z \in M$ under the simultaneous action of both groups, write $H_{1, z}$ and $H_{2, z}$ for the projections of $G_{12, z}$ to the groups $G_{1}$ and $G_{2}$.

Then

(i) $H_{1, z}$ is contained in $G_{1, \Phi_{1}(z)}$, the stabilizer of the image of $z$ under the coadjoint action (i.e., $G_{1, z} \subseteq H_{1, z} \subseteq G_{1, \Phi_{1}(z)}$ ), and

(ii) if, furthermore, the level sets of $\Phi_{1}$ are actually $G_{2}$-orbits, then $H_{1, z}=$ $G_{1, \Phi_{1}(z)}$.

The same statements hold for the indices 1 and 2 interchanged.

Proof. (i) Take $g \in H_{1, z}$, i. e., there exists $g^{\prime} \in G_{2}$ such that $\left(g, g^{\prime}\right) \cdot z=z$. From this one obtains

$$
\Phi_{1}(z)=\Phi_{1}\left(\left(g, g^{\prime}\right) \cdot z\right)=g \cdot \Phi_{1}\left(\left(e, g^{\prime}\right) \cdot z\right)=g \cdot \Phi_{1}(z)
$$

where $G_{1}$-equivariance and $G_{2}$-invariance of $\Phi_{1}$ have been used.

(ii) Now take $g \in G_{1, \Phi_{1}(z)}$, then $\Phi_{1}(g \cdot z)=\Phi_{1}(z)$, i. e., both $z$ and $g \cdot z$ lie in a level set of $\Phi_{1}$, which is by assumption a $G_{2}$-orbit. Thus there exists $g^{\prime} \in G_{2}$ such that $\left(g, g^{\prime}\right) \cdot z=z$, which was to be shown.

Of course, interchanging both actions does not alter the proof.

The particular orbit structure that we have described yields now a correspondence between the orbits in the images of the moment maps $\Phi_{1}$ and $\Phi_{2}$.

Theorem 2.8. Let commuting hamiltonian proper actions of the connected Lie groups $G_{1}$ and $G_{2}$ with equivariant moment maps $\Phi_{1}$ and $\Phi_{2}$ be given on the symplectic manifold $(M, \omega)$.

If the symplectic Howe condition is satisfied, then: 
(i) There is a bijection $\Lambda: \Phi_{1}(M) / G_{1} \rightarrow \Phi_{2}(M) / G_{2}$, given by

$$
\Lambda\left(\mathcal{O}_{\alpha_{1}}\right)=\Phi_{2}\left(\Phi_{1}^{-1}\left(\mathcal{O}_{\alpha_{1}}\right)\right)
$$

where $\alpha_{1} \in \Phi_{1}(M)$, and $\mathcal{O}_{\alpha_{1}}=\operatorname{Ad}^{*}\left(G_{1}\right) \alpha_{1}$ is seen as an element of $\Phi_{1}(M) / G_{1}$. To each pair $\left(\mathcal{O}_{\alpha_{1}}, \Lambda\left(\mathcal{O}_{\alpha_{1}}\right)\right)$ belongs a unique orbit $\left(G_{1} \times G_{2}\right) \cdot z$ in $M$ given by $\Phi_{1}^{-1}\left(\mathcal{O}_{\alpha_{1}}\right)=\Phi_{2}^{-1}\left(\mathcal{O}_{\alpha_{2}}\right)$. We say that orbits $\left(G_{1} \times G_{2}\right) \cdot z$, $\mathcal{O}_{\alpha_{1}}$ and $\mathcal{O}_{\alpha_{2}}$ are in correspondence if $\mathcal{O}_{\alpha_{2}}=\Lambda\left(\mathcal{O}_{\alpha_{1}}\right)$ and $\left(G_{1} \times G_{2}\right) \cdot z=$ $\Phi_{1}^{-1}\left(\mathcal{O}_{\alpha_{1}}\right)=\Phi_{2}^{-1}\left(\mathcal{O}_{\alpha_{2}}\right)$.

(ii) If $\Phi_{1}$ and $\Phi_{2}$ are open maps, then $\Lambda$ is a homeomorphism. The same conclusion holds if the groups $G_{1}$ and $G_{2}$ both are compact and $\Phi_{1}$ and $\Phi_{2}$ both are closed maps. Finally, the same conclusion holds if for $i+j=3$, $G_{i}$ is compact and $\Phi_{i}$ is closed, and $\Phi_{j}$ is open.

(iii) Write $M_{\alpha_{1}}=\Phi_{1}^{-1}\left(\alpha_{1}\right) / G_{1, \alpha_{1}}$ and $M_{\alpha_{2}}=\Phi_{2}^{-1}\left(\alpha_{2}\right) / G_{2, \alpha_{2}}$ for the respective point reduced spaces at $\alpha_{1} \in \mathfrak{g}_{1}^{*}$ and $\alpha_{2} \in \mathfrak{g}_{2}^{*}$. These spaces can be described as coadjoint orbits of the other action, i. e., there are, resp., $G_{2^{-}}$and $G_{1^{-}}$ equivariant symplectomorphisms

$$
M_{\alpha_{1}} \rightarrow \Lambda\left(\mathcal{O}_{\alpha_{1}}\right) \quad \text { and } \quad M_{\alpha_{2}} \rightarrow \Lambda^{-1}\left(\mathcal{O}_{\alpha_{2}}\right) .
$$

(iv) The symplectic reduced space $M_{\left(\alpha_{1}, \alpha_{2}\right)}$ for the joint action of $G_{1} \times G_{2}$ is either a point (if $\mathcal{O}_{\alpha_{1}}$ and $\mathcal{O}_{\alpha_{2}}$ are in correspondence) or empty otherwise.

Proof. In order to simplify indices, some statements will only be proved for one action if the other case is analogous.

(i) By Prop. 2.6, we know that for any $z \in M$, the level sets of both moment maps are orbits: $\Phi_{i}^{-1}\left(\Phi_{i}(z)\right)=G_{j} \cdot z(i+j=3)$. This implies that the preimage of any coadjoint orbit in either moment image $\Phi_{i}(M)(i=1,2)$ is exactly one orbit of the joint action of $G_{1} \times G_{2}$ on $M$, i. e.,

$$
\Phi_{i}^{-1}\left(\operatorname{Ad}^{*}\left(G_{i}\right) \Phi_{i}(z)\right)=\left(G_{1} \times G_{2}\right) \cdot z,
$$

from which it follows that $\Lambda$ is well-defined and bijective.

(ii) Note that all maps in the diagram

$$
\Phi_{1}(M) / G_{1} \stackrel{\pi_{1}}{\longleftarrow} \Phi_{1}(M) \stackrel{\Phi_{1}}{\longleftarrow} M \stackrel{\Phi_{2}}{\longrightarrow} \Phi_{2}(M) \stackrel{\pi_{2}}{\longrightarrow} \Phi_{2}(M) / G_{2}
$$

are continuous, and $\pi_{1}$ and $\pi_{2}$ are always open as well.

Let us denote $\Lambda: \Phi_{1}(M) / G_{1} \rightarrow \Phi_{2}(M) / G_{2}$ by $\Lambda_{12}$ and its set-theoretic inverse by $\Lambda_{21}$. If $\Phi_{1}$ is open, then for $U$ open in $\Phi_{2}(M) / G_{2}$ we have that $\Lambda_{21}(U)=\left(\pi_{1} \circ \Phi_{1}\right)\left(\Phi_{2}^{-1}\left(\pi_{2}^{-1}(U)\right)\right)$ is an open set, i. e., $\Lambda_{12}$ is continuous. Similarly, if $G_{1}$ is compact and $\Phi_{1}$ is closed, we have for $A$ closed in $\Phi_{2}(M) / G_{2}$ that $\Lambda_{21}(A)$ is closed as well, i. e., again $\Lambda_{12}$ is continuous.

The conclusions of (ii) follow easily. 
(iii) Let $z \in M$ and $\alpha_{2}=\Phi_{2}(z)$ its value under the moment map of the second action. Consider the restricted map $\Phi_{2 \mid G_{2} \cdot z}: G_{2} \cdot z \rightarrow \mathcal{O}_{\alpha_{2}}$, and recall that $G_{2} \cdot z=\Phi_{1}^{-1}\left(\alpha_{1}\right)$ for $\alpha_{1}=\Phi_{1}(z)$. Recall from Lemma 2.7 that $G_{1, \alpha_{1}}=$ $\left\{h \in G_{1} \mid \exists g \in G_{2}:(h, g) \cdot z=z\right\}$. This group acts on $G_{2} \cdot z$ and one has $M_{\alpha_{1}}=G_{2} \cdot z / G_{1, \alpha_{1}}$. Thus $\Phi_{2 \mid G_{2} \cdot z}$ induces

$$
\tilde{\Phi}_{2}: M_{\alpha_{1}} \rightarrow \mathcal{O}_{\alpha_{2}}
$$

which inherits from $\Phi_{2}$ smoothness and $G_{2}$-equivariance. It is clearly surjective and we now show that it is injective: Take $\alpha \in \mathcal{O}_{\alpha_{2}}, \tilde{z}_{1}, \tilde{z}_{2} \in G_{2} \cdot z / G_{1, \alpha_{1}}$ so that $\alpha=\tilde{\Phi}_{2}\left(\tilde{z}_{1}\right)=\tilde{\Phi}_{2}\left(\tilde{z}_{2}\right)$. Now fixing preimages $z_{1}, z_{2} \in G_{2} \cdot z$ of $\tilde{z}_{1}, \tilde{z}_{2}$, they have the property $\Phi_{2}\left(z_{1}\right)=\Phi_{2}\left(z_{2}\right)$, and for some $h \in G_{1}$, $z_{2}=h \cdot z_{1}$ holds because the level sets of $\Phi_{2}$ are $G_{1}$-orbits. However, $z_{2}=g \cdot z_{1}$ for some $g \in G_{2}$, which implies by part (ii) of Lemma 2.7 that $h \in G_{1, \alpha_{1}}$. Therefore, $\tilde{z}_{1}=\tilde{z}_{2}$, and $\tilde{\Phi}_{2}$ is a bijection. Applying Sard's Theorem, one notices that a smooth equivariant bijection between finite-dimensional homogeneous spaces has a smooth inverse. Thus $\tilde{\Phi}_{2}$ is a $G_{2}$-equivariant diffeomorphism and it remains to show that $\tilde{\Phi}_{2}$ is a symplectomorphism. Denote by $i_{G_{2} \cdot z}: G_{2} \cdot z \rightarrow M$ the inclusion of $G_{2} \cdot z=\Phi_{1}^{-1}\left(\alpha_{1}\right)$ into the ambient manifold. We observe that the equivariance properties of $\Phi_{2}$ imply $i_{G_{2} \cdot z}^{*} \omega=\left(\Phi_{2 \mid G_{2} \cdot z}\right)^{*} \omega^{\mathcal{O}_{\alpha_{2}}}$, where $\omega^{\mathcal{O}_{\alpha_{2}}}$ is the KKS symplectic form (see, e.g., [OR04], Thm. 4.5.31). But the symplectic form $\omega^{M_{\alpha_{1}}}$ on $M_{\alpha_{1}}$ is defined such that it also pulls back to $i_{G_{2} \cdot z}^{*} \omega=p^{*} \omega^{M_{\alpha_{1}}}$, via the quotient map $p: G_{2} \cdot z \rightarrow G_{2} \cdot z / G_{1, \alpha_{1}}$. Consequently we have $p^{*} \omega^{M_{\alpha_{1}}}=\left(\Phi_{2 \mid G_{2} \cdot z}\right)^{*} \omega^{\mathcal{O}_{\alpha_{2}}}=p^{*}\left(\tilde{\Phi}_{2}^{*} \omega^{\mathcal{O}_{\alpha_{2}}}\right)$. Since $p$ is a surjective submersion, the coincidence of the pullbacks (to $G_{2} \cdot z$ ) implies that $\omega^{M_{\alpha_{1}}}=\tilde{\Phi}_{2}^{*} \omega^{\mathcal{O}_{\alpha_{2}}}$, which was to be shown.

(iv) Let $\Phi=\Phi_{1} \oplus \Phi_{2}$. Take $\alpha_{1} \in \mathfrak{g}_{1}^{*}$ and $\alpha_{2} \in \mathfrak{g}_{2}^{*}$. Then

$$
\Phi^{-1}\left(\mathcal{O}_{\alpha_{1}} \times \mathcal{O}_{\alpha_{2}}\right)
$$

is empty if $\mathcal{O}_{\alpha_{2}} \neq \Lambda\left(\mathcal{O}_{\alpha_{1}}\right)$. Otherwise, for any $z \in M$ such that $\Phi_{1}(z) \in \mathcal{O}_{\alpha_{1}}$ and $\Phi_{2}(z) \in \mathcal{O}_{\alpha_{2}}$, holds

$$
\Phi^{-1}\left(\mathcal{O}_{\alpha_{1}} \times \mathcal{O}_{\alpha_{2}}\right) /\left(G_{1} \times G_{2}\right) \cong\left(G_{1} \times G_{2}\right) \cdot z /\left(G_{1} \times G_{2}\right) .
$$

This quotient is, of course, a point.

Remark 2.9. (1) Statement (i) is a special case of the singular symplectic leaf correspondence of Thm.11.4.4 in [OR04], but here obtained from the symplectic Howe condition on the pair of groups actions, in a spirit close to the non-singular correspondence of Thm.11.1.9 of the same reference.

(2) Take any $z \in M$ and $\alpha_{1}=\Phi_{1}(z)$. Then the global ineffectivity of the $G_{1, \alpha_{1}}$-action on the $\Phi_{1}$-level containing $z$ is $I_{1, \alpha_{1}}=\left\{g_{1} \in G_{1, \alpha_{1}} \mid g_{1} \cdot z^{\prime}=\right.$ 
$\left.z^{\prime} \forall z^{\prime} \in \Phi_{1}^{-1}\left(\alpha_{1}\right)\right\}$, i. e., the intersection of the stabilizers of all points in the level set. Here, $\Phi_{1}^{-1}\left(\alpha_{1}\right)=G_{2} \cdot z$, hence $G_{1, z^{\prime}}=G_{1, z} \subseteq I_{1, \alpha_{1}}$ for all $z^{\prime} \in \Phi_{1}^{-1}\left(\alpha_{1}\right)$, so $I_{1, \alpha_{1}}=G_{1, z}$. Therefore, the proper action of $G_{1, \alpha_{1}}$ on $\Phi_{1}^{-1}\left(\alpha_{1}\right)$ factorizes over a free and proper action of $G_{1, \alpha_{1}} / I_{1, \alpha_{1}}$, and thus the quotient is a smooth manifold.

(3) Part (iv) of the preceding theorem implies that the $\left(G_{1} \times G_{2}\right)$-action on $M$ is multiplicity-free, i. e., $C^{\infty}(M)^{G_{1} \times G_{2}}$ is a commutative Poisson algebra (compare [GS84] and [HW90]). This can also be directly deduced from the definition of a symplectic Howe pair and Lemma 2.2.

\section{Prequantization of Symplectic Howe Pairs}

We are now going to show that the orbit correspondence which was constructed above behaves well under geometric prequantization. The first step is to show that the integrality of coadjoint orbits is preserved under the correspondence. To achieve this, we make use of having proved that the reduced spaces coincide in our setting with coadjoint orbits. Let us recall the following fact (Thm. 3.2 of [GS82]).

Theorem 3.1. Let $G$ be a compact connected Lie group and $(M, \omega)$ be a prequantizable symplectic manifold with hamiltonian $G$-action, equivariant moment map $\Phi$ and line bundle $L(M)$ equipped with a connection $\nabla$ on $L(M)$ whose curvature coincides with $\omega$. If the $G$-action on the level set $\Phi^{-1}(0)$ is free, then the reduced space $M_{0}=\Phi^{-1}(0) / G$ is a manifold and there exists a unique line bundle $L\left(M_{0}\right)$ over $M_{0}$ such that

$$
\pi^{*} L\left(M_{0}\right)=i^{*} L(M) \text { and } \pi^{*} \nabla_{0}=i^{*} \nabla,
$$

where $\pi: \Phi^{-1}(0) \rightarrow \Phi^{-1}(0) / G$ is the quotient map and $i: \Phi^{-1}(0) \rightarrow M$ the inclusion.

Notation. If $(M, \omega)$ is a prequantizable symplectic manifold, i. e., $\omega$ is an integral form, we denote by $L(M, \omega)$ a corresponding prequantum line bundle, i. e., a complex line bundle with first de Rham Chern class equal to $[\omega]$. This line bundle is unique if there exist no torsion line bundles on $M$. We may omit $\omega$ if there is no ambiguity about the symplectic form. For a coadjoint orbit $\mathcal{O}_{\alpha}$, we will write $L_{\alpha}=L\left(\mathcal{O}_{\alpha}\right)$, the KKS symplectic form $\omega^{\mathcal{O}_{\alpha}}$ being understood.

By the shifting trick, any reduced space may be regarded as a reduced space at 0. More precisely, one has the following well-known result (see, e. g., Thm. 6.5.2 in [OR04]).

Theorem 3.2. Let $G$ be a compact connected Lie group and $(M, \omega)$ be a prequantizable symplectic manifold with hamiltonian $G$-action and equivariant moment map $\Phi$. If $G_{\alpha}$ acts freely on $\Phi^{-1}(\alpha)$, then the reduced space at $\alpha \in \mathfrak{g}^{*}$, $\Phi^{-1}(\alpha) / G_{\alpha}$, is symplectomorphic to the reduction of $M \times \mathcal{O}_{\alpha}^{-}$at 0 , where $\mathcal{O}_{\alpha}^{-}$ 
denotes the coadjoint orbit through $\alpha$ with the KKS symplectic form multiplied by -1 .

Returning to the setting of Theorem 2.8, we conclude that any reduced space $M_{\alpha_{1}}$ admits a line bundle $L\left(M_{\alpha_{1}}\right)$ if the coadjoint orbit $\mathcal{O}_{\alpha_{1}}$ does. Recall from Remark 2.9(2) that the quotient of $\Phi_{1}^{-1}\left(\alpha_{1}\right)$ by $G_{1, \alpha_{1}}$ is also given as the quotient by the free $\left(G_{1, \alpha_{1}} / I_{1, \alpha_{1}}\right)$-action on $\Phi_{1}^{-1}\left(\alpha_{1}\right)$ and hence $M_{\alpha_{1}}$ is smooth. Therefore, we can adapt Theorem 3.1 to this quotient. Using the symplectomorphism $\tilde{\Phi}_{2}$ : $M_{\alpha_{1}} \rightarrow \mathcal{O}_{\alpha_{2}}$, we obtain the bundle $\left(\tilde{\Phi}_{2}^{-1}\right)^{*} L\left(M_{\alpha_{1}}\right)$ over $\mathcal{O}_{\alpha_{2}}$. As coadjoint orbits of compact connected Lie groups are simply connected, there are no non-trivial flat vector bundles, hence no torsion line bundles over them. Therefore, for any $\alpha_{1}$ such that $\left(\mathcal{O}_{\alpha_{1}}, \omega^{\mathcal{O}_{\alpha_{1}}}\right)$ is an integral symplectic manifold, the bundle $\left(\tilde{\Phi}_{2}^{-1}\right)^{*} L\left(M_{\alpha_{1}}\right)$ is the unique prequantum line bundle over $\mathcal{O}_{\alpha_{2}}$. Thus we have proved:

Proposition 3.3. Let $\mathcal{O}_{\alpha_{1}}$ and $\mathcal{O}_{\alpha_{2}}$ be two coadjoint orbits in correspondence as in Thm.2.8(i) and assume furthermore that $G_{1}$ and $G_{2}$ are compact. Then $\left(\mathcal{O}_{\alpha_{1}}, \omega^{\mathcal{O}_{\alpha_{1}}}\right)$ is an integral symplectic manifold if and only if $\left(\mathcal{O}_{\alpha_{2}}, \omega^{\mathcal{O}_{\alpha_{2}}}\right)$ is an integral symplectic manifold, too.

Remark 3.4. Let us recall that for $G$ a compact connected semisimple Lie group and $\alpha \in \mathfrak{g}^{*}, \mathcal{O}_{\alpha}$ is an integral symplectic manifold if and only if there exists a character $\chi_{\alpha}: G_{\alpha} \rightarrow U(1)$ such that $\left(\chi_{\alpha}\right)_{* e}=2 \pi \mathrm{i} \alpha$. Thus one defines:

Definition 3.5. (1) Let $G$ be a compact connected Lie group and $\alpha \in \mathfrak{g}^{*}$. We call $\alpha$ integral if there exists $\chi_{\alpha}: G_{\alpha} \rightarrow U(1)$ such that $\left(\chi_{\alpha}\right)_{* e}=2 \pi \mathrm{i} \alpha$.

(2) If $\alpha$ is integral, we call $\mathcal{O}_{\alpha}$ an integral orbit.

Remark 3.6. Of course, there is a natural bijection between the set of integral orbits and the set of dominant weights of $G$ (w.r.t. a fixed Weyl chamber $\mathfrak{t}^{+}$).

We observe that integral coadjoint orbits are integral symplectic manifolds but the converse statement is not true in general because of the possible presence of a positive-dimensional centre. Accordingly, we need to refine Proposition 3.3.

Let us from now on assume that the $\left(G_{1} \times G_{2}\right)$-action on $M$ comes with a fixed "linearization on $L$ ", i. e., there is given a fibrewise linear $\left(G_{1} \times G_{2}\right)$-action on $L=L(M, \omega)$ covering the $\left(G_{1} \times G_{2}\right)$-action on $M$. This is no restriction if one accepts to replace $G_{1}$ and $G_{2}$ by finite coverings (see [Dui96], Prop. 15.4).

Theorem 3.7. Let $\mathcal{O}_{\alpha_{1}}$ and $\mathcal{O}_{\alpha_{2}}$ be two coadjoint orbits in correspondence as in Thm. 2.8(i) and assume furthermore that $G_{1}$ and $G_{2}$ are compact. Then $\mathcal{O}_{\alpha_{1}}$ is integral if and only if $\mathcal{O}_{\alpha_{2}}$ is integral.

Proof. Let $\alpha \in \mathfrak{g}_{1}^{*}$ be integral. Since the $G_{2}$-actions on $M$ and $L$ commute with the $G_{1}$-actions, the $G_{2}$-action on $L$ descends canonically to a $G_{2}$-action on the line bundle $L\left(M_{\alpha_{1}}\right)$ covering the natural $G_{2}$-action on $M_{\alpha_{1}}$. 
Using the $G_{2}$-equivariant symplectomorphism $\tilde{\Phi}_{2}:\left(M_{\alpha_{1}}, \omega^{M_{\alpha_{1}}}\right) \rightarrow\left(\mathcal{O}_{\alpha_{2}}, \omega^{\mathcal{O}_{\alpha_{2}}}\right)$ from above, we have the bundle $\left(\tilde{\Phi}_{2}^{-1}\right)^{*} L\left(M_{\alpha_{1}}\right)=L\left(\mathcal{O}_{\alpha_{2}}\right)$ together with a $G_{2^{-}}$ invariant connection $\nabla$ given by pulling back the $G_{2}$-invariant connection induced on $L\left(M_{\alpha_{1}}\right)$ by a $\left(G_{1} \times G_{2}\right)$-invariant connection on $L \rightarrow M$. (See [GS82] for the construction of the connection on $L\left(M_{\alpha_{1}}\right)$.) We thus arrive at the following commuting diagram (where $p$ denotes the bundle projection and $L\left(\mathcal{O}_{\alpha_{2}}\right)_{\alpha_{2}}=$ $\left.p^{-1}\left(\alpha_{2}\right)\right)$ :

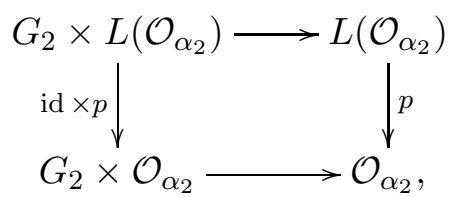

yielding a homomorphism $\chi: G_{2, \alpha_{2}} \rightarrow U\left(L\left(\mathcal{O}_{\alpha_{2}}\right)_{\alpha_{2}}\right)=U(1)$.

It remains to show that $\chi=\chi_{\alpha_{2}}$, i.e., $\chi_{* e}=2 \pi \mathrm{i} \alpha_{2}$. Since the $G_{2}$-action on $L\left(\mathcal{O}_{\alpha_{2}}\right)$ and the connection $\nabla$ come from the reduced bundle with connection $L\left(M_{\alpha_{1}}\right)$ and this comes in turn from a connection on $L \rightarrow M$, we have the usual "Kostant formula" for the fundamental vector fields of the $G_{2}$-action on $L\left(\mathcal{O}_{\alpha_{2}}\right)$. (Compare [Kos70], Thm. 4.5.1.) More precisely, given $\xi \in \mathfrak{g}_{2}, \xi^{L\left(\mathcal{O}_{\alpha_{2}}\right)}=$ $\widetilde{\xi^{\mathcal{O} \alpha_{2}}}+p^{*}\left(\left\langle\Phi_{2}^{\mathcal{O}_{\alpha_{2}}}, \xi\right\rangle\right)(2 \pi \mathrm{i})^{L\left(\mathcal{O}_{\alpha_{2}}\right)}$, where $\xi^{N}$ denotes the fundamental vector field associated to $\xi$ on a $G_{2}$-manifold $N, \widetilde{X}$ denotes the $\nabla$-horizontal lift of a vector field $X$ on $\mathcal{O}_{\alpha_{2}}$ to $L\left(\mathcal{O}_{\alpha_{2}}\right), 2 \pi \mathrm{i} \in \mathrm{i} \mathbb{R} \cong \mathfrak{u}(1)$ also has a fundamental vector field on the bundle by the canonical $U(1)$-action on it, and $\left\langle\Phi_{2}^{\mathcal{O}_{\alpha_{2}}}, \xi\right\rangle$ is the $\xi$-component of the $G_{2}$-moment map on $\mathcal{O}_{\alpha_{2}}$.

For $\xi \in \mathfrak{g}_{2, \alpha_{2}}$, the field $\xi^{L\left(\mathcal{O}_{\alpha_{2}}\right)}$ is now tangent to the $p$-fibre $L\left(\mathcal{O}_{\alpha_{2}}\right)_{\alpha_{2}}$ over $\alpha_{2}$ and equals there $(2 \pi \mathrm{i})^{L\left(\mathcal{O}_{\alpha_{2}}\right)}\left\langle\alpha_{2}, \xi\right\rangle$. Thus $\chi_{* e}: G_{2, \alpha_{2}} \rightarrow U(1)$ is equal to $\left.\frac{\mathrm{d}}{\mathrm{d} t}\right|_{0} e^{2 \pi \mathrm{i}\left\langle\alpha_{2}, \xi\right\rangle t}=2 \pi \mathrm{i}\left\langle\alpha_{2}, \xi\right\rangle$.

\section{Geometric Quantization of Symplectic Howe Pairs}

In this section we show that the symplectic Howe condition for the actions on $M$ implies the representation-theoretic Howe condition for the linear representation on the geometric quantization of $(M, \omega)$ in the Kähler case. Thus we assume throughout this section that $(M, \omega)$ is Kähler, i. e., there is given a complex structure $J$ such that the associated riemannian metric $g(\cdot, \cdot)=\omega(\cdot, J \cdot)$ is positive definite and hermitean. Furthermore we assume that the acting groups are compact and connected and that the moment maps are admissible in the sense of [Sja95] (p. 109).

Definition 4.1. Let $G$ be a compact connected Lie group acting holomorphically on a Kähler manifold $M$ with equivariant moment map $\Phi$. Choose a $G$-invariant inner product on $\mathfrak{g}$ with corresponding norm and define the function $\mu=\|\Phi\|^{2}$. 
Let $F_{t}$ be the gradient flow of $-\mu$. A moment map is called admissible if for every $z \in M$, the path of steepest descent $F_{t}(z)$ through $z(t \geq 0)$ is contained in a compact set.

Remark 4.2. Examples of admissible moment maps are all proper moment maps and the moment map of the natural linear $U(n)$-action on $\mathbb{C}^{n}$.

One then has the following result (Thm.2.20 of [Sja95]).

Theorem 4.3. Let $G$ be a compact connected Lie group acting holomorphically on a Kähler manifold $M$ with admissible equivariant moment map $\Phi$. Suppose this action extends to an action of the complexification $G^{\mathbb{C}}$ of $G$. Then for every dominant weight $\alpha$ of $G$, the space of holomorphic sections $\Gamma_{\mathrm{hol}}\left(M_{\alpha}, L_{\alpha}\right)$ of the prequantum line bundle $L_{\alpha}$ over the symplectic reduced space $M_{\alpha}$ is naturally isomorphic to $\operatorname{Hom}_{G}\left(V_{\alpha}, \Gamma_{\text {hol }}(M, L)\right)$, the space of intertwining operators from the irreducible representation $V_{\alpha}$ with highest weight $\alpha$ to the quantization $\Gamma_{\text {hol }}(M, L)$ of $M$.

Remark 4.4. (1) Of course, dominance of a weight (an analytically integral element of $\mathfrak{t}^{*}$ ) is defined w.r.t. the choice of a Weyl chamber. It is introduced in the statement of the preceding theorem only to avoid redundancy since one has $M_{\mathrm{Ad}^{*}(g) \alpha} \cong M_{\alpha}$ and $V_{\mathrm{Ad}^{*}(g) \alpha} \cong V_{\alpha}$. Thus one may write here integral point of $\mathfrak{g}^{*}$ in the sense of Def. 3.5 as well.

(2) The reduced spaces $M_{\alpha}$ inherit, also in the singular case, "sufficient" structure in order to define holomorphic sections of $L_{\alpha} \rightarrow M_{\alpha}$ (compare [Sja95]). By Theorem 2.8 and Remark 2.9(2) the reduced spaces occurring in our "symplectic Howe setting" are always smooth. Thus, in fact, we do not need the results of Sjamaar in its full generality.

(3) If $\alpha$, interpreted as an element of $\mathfrak{g}^{*}$, is not in the moment image $\Phi(M)$, then $\Gamma_{\text {hol }}\left(M_{\alpha}, L_{\alpha}\right)$ is to be interpreted as $\{0\}$. Theorem 4.3 then says that $V_{\alpha}$ does not occur in the $G$-decomposition of $\Gamma_{\text {hol }}(M, L)$.

Take now the simultaneous $G_{1} \times G_{2}$-action introduced in the previous section. By the Borel-Weil theorem, the geometric quantizations, $V_{\alpha_{i}} \cong \Gamma_{\text {hol }}\left(\mathcal{O}_{\alpha_{i}}, L_{\alpha_{i}}\right)$ with $\alpha_{i}$ integral, realize the irreducible representations of $G_{i}$, thus $\Gamma_{\text {hol }}\left(\mathcal{O}_{\alpha_{1}} \times\right.$ $\left.\mathcal{O}_{\alpha_{2}}, L_{\alpha_{1}} \otimes L_{\alpha_{2}}\right)$ the irreducibles for $G_{1} \times G_{2}$. Here $L_{\alpha_{1}} \otimes L_{\alpha_{2}} \rightarrow M_{1} \times M_{2}$ is given as $p_{1}^{*}\left(L_{\alpha_{1}}\right) \otimes p_{2}^{*}\left(L_{\alpha_{2}}\right)$ with $p_{j}: M_{1} \times M_{2} \rightarrow M_{j}$ denoting the $j$-th projection for $j=1,2$. Now,

$$
\begin{aligned}
\operatorname{Hom}_{G_{1} \times G_{2}}\left(\Gamma _ { \mathrm { hol } } \left(\mathcal{O}_{\alpha_{1}} \times \mathcal{O}_{\alpha_{2}}, L_{\alpha_{1}}\right.\right. & \left.\left.\otimes L_{\alpha_{2}}\right), \Gamma_{\text {hol }}(M, L)\right) \\
& \cong \Gamma_{\mathrm{hol}}\left(\Phi^{-1}\left(\mathcal{O}_{\alpha_{1}} \times \mathcal{O}_{\alpha_{2}}\right) /\left(G_{1} \times G_{2}\right), L_{\left(\alpha_{1}, \alpha_{2}\right)}\right) .
\end{aligned}
$$

By Thm.2.8(iv), the reduced space may be empty (if the coadjoint orbits are not in correspondence), hence the space of sections $\Gamma_{\text {hol }}\left(\Phi^{-1}\left(\mathcal{O}_{\alpha_{1}} \times \mathcal{O}_{\alpha_{2}}\right) /\left(G_{1} \times\right.\right.$ $\left.\left.G_{2}\right), L_{\left(\alpha_{1}, \alpha_{2}\right)}\right)$ is trivial; otherwise the reduced space is a point and the space 
of sections is simply $\mathbb{C}$. So, one concludes for the multiplicities of $G_{1} \times G_{2^{-}}$ representations in the quantization of $M$ :

$$
\operatorname{dim} \operatorname{Hom}_{G_{1} \times G_{2}}\left(\Gamma_{\text {hol }}\left(\mathcal{O}_{\alpha_{1}} \times \mathcal{O}_{\alpha_{2}}, L_{\alpha_{1}} \otimes L_{\alpha_{2}}\right), \Gamma_{\text {hol }}(M, L)\right) \leq 1,
$$

the equal sign being true if and only if $\mathcal{O}_{\alpha_{2}}=\Lambda\left(\mathcal{O}_{\alpha_{1}}\right)$.

Interpreting the line bundles $L_{\alpha_{1}}, L_{\alpha_{2}}$ and $L_{\alpha_{1}} \otimes L_{\alpha_{2}}$ as sheaves and their holomorphic sections as their zeroth cohomology group, one concludes from a standard Künneth formula (compare [SW59] and [Kau67]) that

$$
\Gamma_{\mathrm{hol}}\left(\mathcal{O}_{\alpha_{1}} \times \mathcal{O}_{\alpha_{2}}, L_{\alpha_{1}} \otimes L_{\alpha_{2}}\right) \cong \Gamma_{\mathrm{hol}}\left(\mathcal{O}_{\alpha_{1}}, L_{\alpha_{1}}\right) \otimes \Gamma_{\mathrm{hol}}\left(\mathcal{O}_{\alpha_{2}}, L_{\alpha_{2}}\right) .
$$

Recall that by 2.8(iii), the coadjoint $G_{2}$-orbit corresponding via $\Lambda$ to $\mathcal{O}_{\alpha_{1}} \in$ $\Phi_{1}(M) / G_{1}$ is symplectomorphic to the orbit reduced space of $\mathcal{O}_{\alpha_{1}}$, i. e.,

$$
\Lambda\left(\mathcal{O}_{\alpha_{1}}\right) \cong \Phi_{1}^{-1}\left(\mathcal{O}_{\alpha_{1}}\right) / G_{1}=M_{\alpha_{1}} .
$$

This implies that the multiplicity space of one action is an irreducible representation of the other action, i. e., for $\mathcal{O}_{\alpha_{2}}=\Lambda\left(\mathcal{O}_{\alpha_{1}}\right)$, one has $G_{2}$-equivariantly

$$
\operatorname{Hom}_{G_{1}}\left(V_{\alpha_{1}}, \Gamma_{\text {hol }}(M, L)\right) \cong \Gamma_{\text {hol }}\left(M_{\alpha_{1}}, L\left(M_{\alpha_{1}}\right)\right) \cong \Gamma_{\text {hol }}\left(\mathcal{O}_{\alpha_{2}}, L_{\alpha_{2}}\right) .
$$

Let us identify in the sequel (for $k=1,2) \widehat{G_{k}}$, the set of equivalence classes of irreducible complex representations of finite dimension of $G_{k}$, with $\left(\mathfrak{t}_{k}\right)_{\mathbb{Z}}^{+}$the integral points in a fixed Weyl chamber $\mathfrak{t}_{k}^{+}$in the dual $\mathfrak{t}_{k}^{*}$ of a maximal abelian subalgebra $\mathfrak{t}_{k} \subseteq \mathfrak{g}_{k}$.

The preceding statements can be summarized as follows.

Theorem 4.5. Let $G_{1}$ and $G_{2}$ be compact connected Lie groups acting by holomorphic transformations on a Kähler manifold $M$ such that the actions extend to actions of the respective complexified groups. Suppose that the actions of $G_{1}$ and $G_{2}$ commute and are hamiltonian with admissible equivariant moment maps $\Phi_{1}$ and $\Phi_{2}$. Denote by $L$ the prequantum line bundle over $M$.

Assume the symplectic Howe condition to be satisfied. Then:

$$
\Gamma_{\text {hol }}(M, L) \cong \widehat{\bigoplus}_{\alpha_{1} \in \Phi_{1}(M) \cap \widehat{G_{1}}} \Gamma_{\text {hol }}\left(\mathcal{O}_{\alpha_{1}}, L_{\alpha_{1}}\right) \otimes \Gamma_{\text {hol }}\left(\mathcal{O}_{\alpha_{2}}, L_{\alpha_{2}}\right)
$$

where $\Lambda$ is the orbit correspondence map and $\mathcal{O}_{\alpha_{2}}=\Lambda\left(\mathcal{O}_{\alpha_{1}}\right)$.

Remark 4.6. (1) In the preceding theorem the symbol $\widehat{\bigoplus}$ of course signifies the completion of the algebraic direct sum in the Fréchet topology of the space of holomorphic sections of $L$.

(2) Since $\Lambda$, viewed as a map from $\Phi_{1}(M) \cap \widehat{G_{1}}$ to $\widehat{G_{2}}$, is injective the representation $\Gamma_{\text {hol }}(M, L)$ of the pair $\left(G_{1}, G_{2}\right)$ satisfies the representation-theoretic Howe condition $(*)$ recalled in the introduction. 
(3) Let $(M, \omega)$ be a compact complex manifold together with an integral Kähler form and $L \rightarrow M$ a prequantizing holomorphic line bundle. Let furthermore the connected compact Lie groups $G_{1}$ and $G_{2}$ act by holomorphic transformations and in a hamiltonian fashion on $M$. If this pair of actions satisfies the symplectic Howe condition, the preceding theorem applies, i. e., their induced linear representation on the "holomorphic quantization" $\Gamma_{\text {hol }}(M, L)$ is equipped with a Howe duality.

\section{$5 \quad$ Examples}

In this section we give some simple but instructive examples of pairs of symplectic actions fulfilling the symplectic Howe condition. In each case, we explicitly give the orbit correspondence map and the Howe duality map associated to the linear representation of the pair of groups on the geometric quantization space.

\section{$5.1(U(n), U(m))$ on $\operatorname{Mat}(n \times m ; \mathbb{C})$}

Let $M=\operatorname{Mat}(n \times m ; \mathbb{C})$ be the $n \times m$ matrices with complex entries equipped with the symplectic structure $\omega(A, B)=\operatorname{Im} \operatorname{tr}\left(\bar{A}^{\mathrm{T}} B\right)$ and the natural actions of $G_{1}=U(n)$ and $G_{2}=U(m): U(n) \times U(m) \times M \rightarrow M,((U, V), z) \mapsto U z V^{-1}$. Assume that $n \geq m \geq 1$. The (equivariant) moment maps corresponding to these actions are given by $\Phi_{1}^{\xi}(z)=-\frac{1}{2} \operatorname{Im} \operatorname{tr}\left(\xi z \bar{z}^{\mathrm{T}}\right)$ (for $\left.\xi \in \mathfrak{u}(n)\right)$ and $\Phi_{2}^{\eta}(z)=$ $\frac{1}{2} \operatorname{Im} \operatorname{tr}\left(\eta \bar{z}^{\mathrm{T}} z\right)$ (for $\left.\eta \in \mathfrak{u}(m)\right)$. Direct inspection shows that any level set of $\Phi_{1}$ is exactly one $U(m)$-orbit. Using standard facts from invariant theory and a theorem of G. Schwarz (see [Sch75], Thm.1) one obtaines that the space of pullbacks of smooth functions on $\mathfrak{g}_{1}^{*}$ under $\Phi_{1}$ coincides with the algebra of $G_{2}$-invariant smooth functions on $M$, and thus by Lemma 2.2 with the centralizer of the $\Phi_{2^{-}}$ pullback of $C^{\infty}\left(\mathfrak{g}_{2}^{*}\right)$ in the Poisson algebra $C^{\infty}(M)$. The analogous statements hold if the roles of the two groups are reversed. Therefore, the symplectic Howe condition is satisfied.

In order to state the orbit correspondence of Thm. 2.8 explicitly, recall that any $z \in M$ can be written $z=U \Sigma V$ for

$$
\Sigma=\Sigma\left(\sigma_{1}, \ldots, \sigma_{m}\right)=\left(\begin{array}{cccc}
\sigma_{1} & 0 & \cdots & 0 \\
0 & \sigma_{2} & \cdots & 0 \\
\vdots & \vdots & \ddots & \vdots \\
0 & 0 & \cdots & \sigma_{m} \\
0 & 0 & \cdots & 0 \\
\vdots & \vdots & \vdots & \vdots
\end{array}\right) \in M
$$

where $\sigma_{1} \geq \sigma_{2} \geq \ldots \geq \sigma_{m} \geq 0$ and $U \in U(n), V \in U(m)$ are determined up to an element of the stabilizer of $\Sigma$. The set $S=\left\{\sigma=\left(\sigma_{1}, \ldots, \sigma_{m}\right) \mid \sigma_{1}>\sigma_{2}>\ldots>\right.$ $\left.\sigma_{m}>0\right\}$ parametrizes thus all $U(n) \times U(m)$-orbits of maximal dimension and 
its closure $\bar{S}$ all $U(n) \times U(m)$-orbits. Identifying the adjoint and the coadjoint action of a unitary group via the usual trace form isomorphism $(\zeta \mapsto \operatorname{tr}(\cdot \zeta))$ we get $\widetilde{\Phi}_{1}(z)=\frac{\mathrm{i}}{2} z \bar{z}^{\mathrm{T}}$ and $\widetilde{\Phi}_{2}(z)=-\frac{\mathrm{i}}{2} \bar{z}^{\mathrm{T}} z$ fulfilling $\Phi_{1}^{\xi}(z)=\operatorname{tr}\left(\xi \cdot \widetilde{\Phi}_{1}(z)\right)$ and $\Phi_{2}^{\eta}(z)=\operatorname{tr}\left(\eta \cdot \widetilde{\Phi}_{2}(z)\right)$. Denoting furthermore the diagonal element of $\mathfrak{u}(N)$ with entries $\mathrm{i}\left(\lambda_{1}, \ldots, \lambda_{N}\right)$ by $\left[\lambda_{1}, \ldots, \lambda_{N}\right]$ or simply by $[\lambda]$ we have here the following orbit correspondence map:

$$
\Lambda: \mathcal{O}_{\left[\frac{1}{2} \sigma_{1}^{2}, \ldots, \frac{1}{2} \sigma_{m}^{2}, 0, \ldots, 0\right]}^{U(n)} \mapsto \mathcal{O}_{\left[-\frac{1}{2} \sigma_{1}^{2}, \ldots,-\frac{1}{2} \sigma_{m}^{2}\right]}^{U(m)} .
$$

Observe that orbits of any dimension are included in this statement and no restriction to the principal orbit type is necessary. For the sake of brevity we write $[\alpha, 0]$ for $\left[\alpha_{1}, \ldots, \alpha_{m}, 0, \ldots, 0\right]$ in the sequel.

Now, we may apply Thm. 4.5 and obtain, since the prequantum line bundle $L$ over $M$ is holomorphically trivial,

$$
\begin{aligned}
& \operatorname{Hol}(M)=\Gamma_{\text {hol }}(M, L)=\widehat{\bigoplus}_{\alpha \in \bar{S} \text { integral }} \Gamma_{\text {hol }}\left(\mathcal{O}_{[\alpha, 0]}^{U(n)}, L_{[\alpha, 0]}\right) \otimes \Gamma_{\text {hol }}\left(\mathcal{O}_{[-\alpha]}^{U(m)}, L_{[-\alpha]}\right) \\
& \cong \bigoplus_{\alpha \in \bar{S} \text { integral }} V_{[\alpha, 0]} \otimes\left(V_{[\alpha]}\right)^{*},
\end{aligned}
$$

where for $\lambda=\left(\lambda_{1}, \ldots, \lambda_{N}\right)$ (with $\lambda_{1}, \ldots \lambda_{n}$ integral and $\lambda_{1} \geq \lambda_{2} \geq \ldots \geq$ $\left.\lambda_{N}\right), V_{[\lambda]}$ is the irreducible $U(N)$-representation associated to the orbit through $\mathrm{i}\left(\lambda_{1}, \ldots, \lambda_{N}\right)$ either by the orbit method or equivalently by the highest-weight procedure. The ensuing Howe duality map, $V_{[\alpha, 0]} \mapsto\left(V_{[\alpha]}\right)^{*}$, is then essentially a restatement of the well-known $G L(n)-G L(m)$-duality (compare, e.g., Thm. 5.2.7 in [GW98]) on the space of holomorphic polynomials on $M$.

\section{$5.2(G, G)$ on $T^{*} G$}

Given a Lie group $G$, the left and right action of $G$ on itself of course lift to its cotangent bundle $T^{*} G$. Both actions are free and proper, but this is not in general true for the product. Trivializing $T^{*} G$ via the right action, the left resp. right action reads as follows: $(g, \alpha) \mapsto\left(h g, \mathrm{Ad}^{*}(h) \alpha\right)$ and $(g, \alpha) \mapsto\left(g h^{-1}, \alpha\right)$, respectively. Furthermore, the moment maps are then given by $\Phi_{1}:(g, \alpha) \mapsto \alpha$ and $\Phi_{2}:(g, \alpha) \mapsto-\operatorname{Ad}^{*}\left(g^{-1}\right) \alpha$ for the left and right action, respectively. Obviously, the $\Phi_{1}$-fibres are right- $G$-orbits and the $\Phi_{2}$-fibres left- $G$-orbits. For connected $G$ the symplectic Howe condition can easily be verified using Lemma 2.2; the orbit correspondence is $\Lambda: \mathcal{O}_{\alpha} \mapsto \mathcal{O}_{-\alpha}$.

Restricting now to the case that $G$ is compact and connected, $T^{*} G$ can be identified with the affine Kähler manifold $G^{\mathbb{C}}$ (see, e.g., Sect. 3 in [Hal97]) and the holomorphic geometric quantization $\operatorname{Hol}\left(G^{\mathbb{C}}\right)$ has the same $(G \times G)$-finite vectors as the representation on $L^{2}(G)$. In this situation, Thm. 4.5 specializes thus to the Peter-Weyl theorem. 


\section{$5.3(U(1), U(n))$ on $\mathbb{P}_{n}(\mathbb{C})$}

The standard $U(n+1)$-action on $\mathbb{P}_{n}(\mathbb{C})$ preserves the complex structure and the scaled Fubini-Study Kähler form $k \omega^{\mathrm{FS}}(k \in \mathbb{N} \backslash\{0\})$, and possesses the following equivariant moment map (for $\zeta \in \mathfrak{u}(n+1)$ ):

$$
\Phi^{\zeta}([z])=k \frac{\mathrm{i}}{2 \pi} \frac{\langle z, \zeta \cdot z\rangle}{\langle z, z\rangle} .
$$

The injections $U(n) \rightarrow U(n+1), A \mapsto\left(\begin{array}{ll}1 & 0 \\ 0 & A\end{array}\right)$ and $U(1) \rightarrow U(n+1), e^{2 \pi \mathrm{i} \vartheta} \mapsto$ $\left(\begin{array}{cc}e^{2 \pi \mathrm{i} \vartheta} & 0 \\ 0 & \mathbf{1}_{n}\end{array}\right)$ yield a pair of commuting symplectic actions with induced moment maps. A study of invariant functions on $\mathbb{P}_{n}(\mathbb{C})$ shows that the action of the pair $(U(1), U(n))$ satisfies the symplectic Howe condition.

Fixing $\xi_{0}=2 \pi$ i the moment map $\Phi_{1}$ reads as $\Phi^{\xi_{0}} \cdot \xi_{0}^{*} \in \mathfrak{u}(1)^{*}$ and -identifying $\mathfrak{u}(1)^{*}$ with $\mathbb{R}$ via the base consisting of $\xi_{0^{-}}^{*}$ one has that $\Phi_{1}(M)=[-k, 0]$. We also find that $\Phi_{2}(M)=U(n) \cdot S$, where $S=\left\{y \cdot \varphi_{11} \mid 0 \leq y \leq k\right\}$ is a global slice, with $\varphi_{11}(X)=\frac{\mathrm{i}}{2 \pi} X_{11}$ for $X \in \mathfrak{u}(n) \subseteq \operatorname{Mat}(n \times n ; \mathbb{C})$. The orbit correspondence $\Lambda$ is then given by $x \mapsto U(n) \cdot\left((x+k) \varphi_{11}\right)$. Integral points in $\Phi_{1}(M)$ are simply points $m \in[-k, 0] \cap \mathbb{Z}$ with associated representation $e^{2 \pi \mathrm{i} \vartheta} \mapsto e^{2 \pi \mathrm{i} m \vartheta}$, whereas in $\Phi_{2}(M)$ an orbit $U(n) \cdot\left(y \varphi_{11}\right)$ with $y \geq 0$ is integral if and only if $y \in \mathbb{N}$. Obviously, integrality of orbits in the sense of Def. 3.5 is preserved by the orbit correspondence map $\Lambda$.

For $k>0$, the geometric quantization of $\left(\mathbb{P}_{n}(\mathbb{C}), k \omega^{\mathrm{FS}}\right)$ is given by the holomorphic sections module of the $k$-th power of the hyperplane bundle over $\mathbb{P}_{n}(\mathbb{C})$, isomorphic as a $U(n+1)$-representation to $\mathbb{C}_{k}\left[z_{0}, z_{1}, \ldots, z_{n}\right]$, the space of complex homogeneous polynomials of degree $k$ in $n+1$ variables.

As a $(U(1) \times U(n))$-representation this space decomposes as

$$
\bigoplus_{d=0}^{k} \mathbb{C}_{d}\left[z_{0}\right] \otimes \mathbb{C}_{k-d}\left[z_{1}, \ldots, z_{n}\right],
$$

where for $l \in \mathbb{N}, \mathbb{C}_{l}\left[z_{1}, \ldots, z_{n}\right]$ is the space of complex homogeneous polynomials of degree $l$ in $n$ variables, the $U(n)$-representation associated to $U(n) \cdot\left(l \varphi_{11}\right) \in$ $\Phi_{2}(M) / U(n)$, and $\mathbb{C}_{d}\left[z_{0}\right]$ realizes the $U(1)$-representation on $\mathbb{C}$ given by the character $e^{2 \pi \mathrm{i} \vartheta} \mapsto e^{2 \pi \mathrm{i}(-d) \vartheta}$ associated to $-d \in \Phi_{1}(M) \cap \mathbb{Z}$. The representationtheoretic Howe duality map of this $(U(1) \times U(n))$-representation, $\mathbb{C}_{d}\left[z_{0}\right] \mapsto$ $\mathbb{C}_{k-d}\left[z_{1}, \ldots, z_{n}\right]$, is then clearly induced by the orbit correspondence map.

\section{Acknowledgements}

We would like to thank Joachim Hilgert for several helpful discussions related to this work. The second named author would like to thank as well Marcus J. Slupinski for anterior discussions on the notion of Howe duality. 


\section{References}

[Ada87] Jeffrey Adams. Coadjoint orbits and reductive dual pairs. Adv. in Math., 63:138-151, 1987.

[Dui96] Johannes J. Duistermaat. The heat kernel Lefschetz fixed point formula

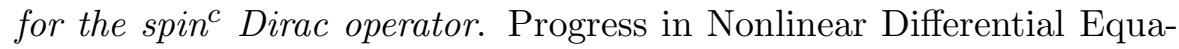
tions and their Applications, 18. Birkhäuser Boston Inc., Boston, MA, 1996.

[GS82] Victor Guillemin and Shlomo Sternberg. Geometric quantization and multiplicities of group representations. Invent. Math., 67:515-538, 1982.

[GS84] Victor Guillemin and Shlomo Sternberg. Multiplicity-free spaces. J. Differential Geometry, 19:31-56, 1984.

[GW98] Roe Goodman and Nolan R. Wallach. Representations and invariants of the classical groups, volume 68 of Encyclopedia of Mathematics and its Applications. Cambridge University Press, Cambridge, 1998.

[Hal97] Brian C. Hall. Phase space bounds for quantum mechanics on a compact Lie group. Comm. Math. Phys., 184(1):233-250, 1997.

[How89] Roger Howe. Remarks on classical invariant theory. Trans. Amer. Math. Soc., 313(2):539-570, 1989.

[HW90] Alan T. Huckleberry and Tilmann Wurzbacher. Multiplicity-free complex manifolds. Math. Ann., 286(1-3):261-280, 1990.

[Kau67] Ludger Kaup. Eine Künnethformel für Fréchetgarben. Math. Z., 97:158-168, 1967.

[KKS78] D. Kazhdan, B. Kostant, and S. Sternberg. Hamiltonian group actions and dynamical systems of Calogero type. Comm. Pure Appl. Math., 31(4):481-507, 1978.

[KL97] Yael Karshon and Eugene Lerman. The centralizer of invariant functions and division properties of the moment map. Illinois J. Math., 41(3):462-487, 1997.

[Kos70] Bertram Kostant. Quantization and unitary representations. I. Prequantization. In Lectures in modern analysis and applications, III, pages 87-208. Lecture Notes in Math., Vol. 170. Springer, Berlin, 1970.

[OR04] Juan-Pablo Ortega and Tudor S. Ratiu. Momentum maps and Hamiltonian reduction, volume 222 of Progress in Mathematics. Birkhäuser Boston Inc., Boston, MA, 2004.

[RN76] Léon Rosenfeld and Jens Rud Nielsen (eds.). The Correspondence Principle (1918-1923), volume 3 of Niels Bohr Collected Works. NorthHolland, Amsterdam, 1976.

[Sch75] Gerald W. Schwarz. Smooth functions invariant under the action of a compact Lie group. Topology, 14:63-68, 1975. 
[Sja95] Reyer Sjamaar. Holomorphic slices, symplectic reduction and multiplicities of representations. Ann. of Math. (2), 141(1):87-129, 1995.

[SW59] Joseph H. Sampson and Gerard Washnitzer. A Künneth formula for coherent algebraic sheaves. Illinois J. Math., 3:389-402, 1959.

[Wei83] Alan Weinstein. The local structure of Poisson manifolds. J. Differential Geom., 18(3):523-557, 1983. 